\title{
Research status and hotspots of economic evaluation in nursing by co-word clustering analysis
}

Yao-Ji Liaoa, Guo-Zhen Gaob,*

aSchool of Nursing, Guangzhou Medical University, Guangzhou, Guangdong, China

${ }^{b}$ Department of Nursing, The First Affiliated Hospital of Guangzhou Medical University, Guangzhou, Guangdong, China

Received: 29 September 2018; Accepted: 6 January 2019; Published: 20 September 2019

Abstract: Objective: The aim of this study is to discover research status and hotspots of economic evaluation (EE) in nursing area using co-word cluster analysis.

Methods: Medical Subject Heading (MeSH) term "cost-benefit analysis" was searched in PubMed and nursing journals were limited by the function of filter. The information of author, country, year, journal, and keywords of collected paper was extracted and exported to Bicomb 2.0 system, where high-frequency terms and other data could be further mined. SPSS 19.0 was used for cluster analysis to generate dendrogram.

Results: In all, 3,020 articles were found and 10,573 MeSH terms were detected; among them, 1,909 were MeSH major topics and generated 42 high-frequency terms. The consequence of dendrogram showed seven clusters, representing seven research hotspots: skin administration, infection prevention, education program, nurse education and management, EE research, neoplasm patient, and extension of nurse function.

Conclusions: Nursing EE research involved multiple aspects in nursing area, which is an important indicator for decision-making. Although the number of papers is increasing, the quality of study is not promising. Therefore, further study may be required to detect nurses' knowledge of economic analysis method and their attitude to apply it into nursing research. More nursing economics course could carry out in nursing school or hospitals.

Keywords: cost-benefit analysis • co-word clustering analysis • economic evaluation • nursing • nursing education

(c) Shanxi Medical Periodical Press.

\section{Introduction}

As people's multiple demands for health care are growing, the discussion about optimization and allocation of nursing resource continues. ${ }^{1}$ How to balance the limited nursing resources with the required quality of care becomes a problem for hospital and government administrators. If they are making a decision to implement only one of the projects or a project costlier than the traditional one, the difficulty would be much bigger. One of the instruments can help in the selection process of alternative policies, programs, projects, or regulations in health economic evaluation (EE). Health $E E$ is defined as the comparative analysis of alternative courses of action in terms of both their costs and consequences. ${ }^{2}$ It is a decision-making process that can

How to cite this article: Liao YJ, Gao GZ. Research status and hotspots of economic evaluation in nursing by co-word clustering analysis. Front Nurs. 2019; 3: 233-240. 
provide comparative data to evaluate "choices," helping policymakers to determine the most cost-effective option for allocation. ${ }^{3}$ Cost-effectiveness analysis, costutility analysis, and cost-benefit analysis are common methods used in EE. To date, the results of nursing EE have been widely used in nursing practice and management, helping nurse to select effective interventions, ${ }^{4}$ evaluating the cost-effectiveness of healthcare program, ${ }^{5}$ and predicting the availability of human resource management plan. ${ }^{6}$ Currently, nursing EE is playing more and more important role in nursing area. More scholars would participate in the study of nursing costbenefit analysis. Therefore, the aim of our study is to apply co-word cluster analysis to discover the research status and hotspots of nursing EE so as to provide more references for further research.

\section{Methods}

\subsection{Search strategy}

PubMed was the most popular and widely used database, comprising more than 28 million citations for biomedical literature from MEDLINE, life science journals, and online books. Medical Subject Heading (MeSH) term was a controlled vocabulary to index articles in MEDLINE. ${ }^{7}$ They were assigned to each document not only based on the abstract and/or the title but also based on the context of the whole document; thus, they contain high-density information from the document. ${ }^{8}$ "CostBenefit Analysis" was first introduced as a MeSH term in 1976. Any papers included terms, such as "Cost Utility Analysis," "Economic Evaluation," and "Cost Effectiveness Analysis," could also be found using "Cost-Benefit Analysis." We did not use other terms like "economic" or "cost" because their results included some studies that just simply calculate the healthcare cost instead of applying EE methods. Therefore, "Cost-Benefit Analysis" was searched as a MeSH term in PubMed up to May 2, 2018. We also used the function of filter to limit the journal category in nursing. All papers we found were included.

\subsection{Data extraction and processing}

The data of title, author, country, language, year, and $\mathrm{MeSH}$ terms of the searching papers were exported in $\mathrm{XML}$ format. The XML file was processed in Bicomb $2.0,{ }^{9}$ which is a text mining system to investigate the co-occurrence of bibliographic data in literature. The frequencies of title, author, country, language, year, and $\mathrm{MeSH}$ terms were accumulated and the connections between each paper were investigated through their MeSH terms. Statistical data were recorded in the system window and could be exported to Excel. To make the results more focused, the following words were combined or transformed, as they have hierarchical relationship based on MeSH Tree Structure. First, "Hospital Costs" and "Drug Cost" were merged into "Health Care Costs." Second, "Health Promotion" was merged into "Health Education." Third, "Education, Nursing, Associate," "Education, Nursing, Baccalaureate," "Education, Nursing, Continuing," "Education, Nursing, Diploma Programs," and "Education, Nursing, Graduate" were all merged into "Education, Nursing." We also delete "Cost-Benefit Analysis" which is a search term that without reflecting any research topics. Demarcation of high- and low-frequency terms was calculated using the formula ${ }^{10}: \mathrm{T}=\left(-1+\sqrt{1+8^{*} / 1}\right) / 2$, where 11 represents the amount of terms whose frequency was 1.

\subsection{Data analysis}

Co-word matrix of high-frequency terms was produced by Bicomb 2.0. Hierarchical cluster analysis in SPSS 19.0 was used to analyze these data. Cluster method chose "Between-group linkage" and measure chose "binary: Ochiai" to generate Dendrogram.

\section{Results}

\subsection{Results of high-frequency terms}

We found 3,020 articles and they were published from 28 different countries, $91 \%$ of them from United States $(1,973)$, England (722), and Canada (53). A total of 368 nursing journals were discovered. The top three were Nursing Economic and The Journal of Nursing Administration with 111 published papers and Infection Control and Hospital Epidemiology with 100. In total, $10,573 \mathrm{MeSH}$ terms were detected in all the papers but $1,909 \mathrm{MeSH}$ major topics were used to produce the highfrequency terms. The threshold value was 41 based on the formula calculation. Therefore, 42 high-frequency terms were obtained, accounting for $29.7 \%$ of the total (Table 1). The term "Education, Nursing" has the highest frequency of 185 and the second is "Nursing Staff, Hospital" of 182.

\subsection{Results of hierarchical cluster analysis}

Dendrogram is shown in Figure 1. Based on the structure of dendrogram and professional knowledge, seven clusters representing seven research topics were classified and named. Details of each topic name and its 


\begin{tabular}{|c|c|c|c|c|c|}
\hline No. & MeSH major topic & Frequency & No. & MeSH major topic & Frequency \\
\hline 1 & Education, Nursing & 185 & 22 & Mass Screening & 61 \\
\hline 2 & Nursing Staff, Hospital & 182 & 23 & Nursing Care & 60 \\
\hline 3 & Health Care Costs & 177 & 24 & Nursing Service, Hospital & 60 \\
\hline 4 & Pressure Ulcer & 117 & 25 & Cross Infection & 58 \\
\hline 5 & Home Care Services & 115 & 26 & State Medicine & 57 \\
\hline 6 & Nurse Practitioners & 112 & 27 & Patient Education as Topic & 56 \\
\hline 7 & Wounds and Injuries & 108 & 28 & Primary Health Care & 55 \\
\hline 8 & Nurse Clinicians & 102 & 29 & Inservice Training & 55 \\
\hline 9 & Quality of Health Care & 89 & 30 & Nurse Administrators & 48 \\
\hline 10 & Nurse's Role & 89 & 31 & Beds & 47 \\
\hline 11 & Community Health Nursing & 87 & 32 & Economics, Nursing & 46 \\
\hline 12 & Bandages & 82 & 33 & Managed Care Programs & 45 \\
\hline 13 & Outcome Assessment (Health Care) & 81 & 34 & Job Description & 44 \\
\hline 14 & Case Management & 72 & 35 & Infection Control & 43 \\
\hline 15 & Health Education & 70 & 36 & Occupational Health Services & 43 \\
\hline 16 & Models, Nursing & 69 & 37 & Attitude of Health Personnel & 42 \\
\hline 17 & Quality Assurance, Health Care & 66 & 38 & Nursing Staff & 42 \\
\hline 18 & Skin Care & 66 & 39 & Delivery of Health Care & 41 \\
\hline 19 & Critical Care & 64 & 40 & Neoplasms & 41 \\
\hline 20 & Personnel Staffing and Scheduling & 64 & 41 & Nursing Research & 41 \\
\hline 21 & Patient Care Team & 62 & 42 & Wound Healing & 41 \\
\hline
\end{tabular}

Table 1. Summary of all high-frequency terms.

terms are shown in Table 2. The smallest group contains three terms and the largest group has 11 terms.

\section{Discussion}

Seven clusters representing seven research topics were determined by statistical data and expertise.

\subsection{Nursing EE on skin administration}

Hospital-acquired skin breakdown usually caused high economic burden on healthcare system. ${ }^{11}$ EE is helpful to discover much cost-effective nursing interventions in skin administration. Hotspots of this class of study focused on the comparison of different intervention and products to prevent pressure ulcers or fasten wound healing. For example, a study showed that nutritional support was cost saving and gained 0.005 quality-adjusted life years compared with standard care to prevent pressure ulcers. ${ }^{12}$ Another study found that a novel soluble beta-glucan gel has a shorter healing time that leads to cost saving over an annual budget cycle of $£ 503$ per patient, compared with methylcellulose dressing. ${ }^{13}$ With a deep understanding of the mechanism of wounds and the discovery of innovative products or equipment, this study will continue.

\subsection{Nursing EE on infection prevention}

Three terms, "cross infection," "infection control," and "mass screening," in this group indicate that the search topic is about infection prevention. Healthcareassociated infections (HAls) are infections that patients get while receiving treatment for medical or surgical conditions. It is estimated that HAl led to costs of more than $\$ 100$ million in one state of United States in 2009. ${ }^{14}$ In a study, seven strategies were found to be available to standard precautions in ICU, but based on the method of EE, the author found universal decolonization was the best choice because it was least expensive but moderately effective. ${ }^{15}$ Mass screening is a way for infection prevention. However, by EE, a study showed us that even though a universal methicillin-resistant Staphylococcus aureus (MRSA) screening may provide potential benefit in preventing MRSA infection, it is relatively costly and may be economically burdensome for a hospital. ${ }^{16}$ 
Pressure Ulcer

Beds

Critical Care

Wounds and Injuries

Skin Care

Bandages

Wound Healing

Cross Infection

Infection Control

Mass Screening

Health Education

Occupational Health Services

Patient Education as Topic

Nursing Service, Hospital

Nurse Administrators

Nursing Staff, Hospital

Personnel Staffing and Scheduling

Education, Nursing

Inservice Training

Attitude of Health Personnel

Nursing Staff

Quality Assurance, Health Care

State Medicine

Nursing Care

Economics, Nursing

Quality of Health Care

Nursing Research

Health Care Costs

Neoplasms

Delivery of Health Care

Nurse Clinicians

Job Description

Nurse Practitioners

Nurse's Role

Primary Health Care

Patient Care Team

Managed Care Programs

Home Care Services

Community Health Nursing

Outcome Assessment (Health Care)

Case Management

Models, Nursing

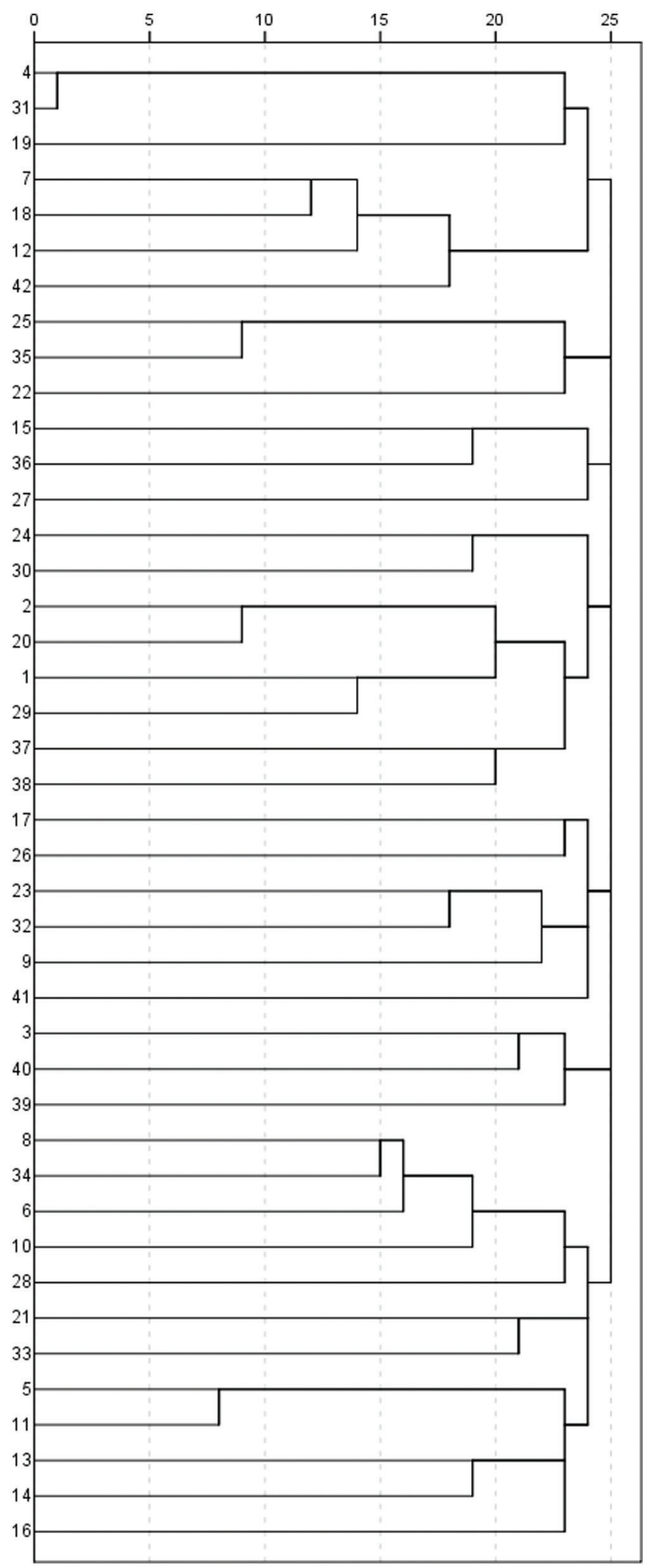

Figure 1. Dendrogram of cluster analysis. 


\begin{tabular}{cll}
\hline No. & Topics & Terms \\
\hline \hline 1 & Skin administration & Pressure Ulcer; Beds; Wounds and Injuries; Critical Care; Skin Care; Bandages; Wound Healing \\
2 & Infection prevention & Cross Infection; Infection Control; Mass Screening \\
3 & Education program & Health Education; Occupational Health Services; Patient Education as Topic \\
4 & Nurse education and management & Nursing Service, Hospital; Nurse Administrators; Nursing Staff, Hospital; Personnel Staffing and \\
& & Scheduling; Education, Nursing; Inservice Training; Attitude of Health Personnel; Nursing Staff \\
5 & Exploration of economic evaluation & Quality Assurance, Health Care; State Medicine; Nursing Care; Economics, Nursing; Quality of \\
& research in nursing field & Health Care; Nursing Research \\
6 & Care for neoplasm patient & Health Care Costs; Neoplasms; Delivery of Health Care \\
7 & Extension of nurse function & Nurse Clinicians; Job Description; Nurse Practitioners; Nurse's Role; Primary Health Care; Patient \\
& & Care Team; Managed Care Programs; Home Care Services; Community Health Nursing; Outcome \\
& & Assessment (Health Care); Case Management; Models, Nursing \\
\hline
\end{tabular}

Table 2. Topics and their terms.

\subsection{Nursing EE on education program}

According to the MeSH terms definition in PubMed, "Health Education" means education that increases the awareness and favorably influences the attitudes and knowledge relating to the improvement of health on a personal or community basis; "Occupational Health Services" means health services for employees, usually provided by the employer at the place of work; and "Patient Education as Topic" means the teaching or training of patients concerning their own health needs. They all conveyed the topic of education on both health person and patient. People with chronic disease and employee in hospital or other place are main study participants. The cost-effectiveness ratios and cost per quality-adjusted life year were commonly used as evaluation indicators. Cost-benefit analysis supports the more widespread implementation of some education program, ${ }^{17}$ and also justify some programs are not cost-effective which should be further developed and modified. ${ }^{18}$

\subsection{Nursing EE on nurse education and management}

The terms in this study group include "Education, Nursing" and "Nursing Staff, Hospital" which rank top two of all key terms, indicating nursing education and management is the most popular research topic in EE. Nursing staff education and training is determined by management department of hospital, so these two kinds of studies would have strong connections. Improving the educational level of nurse means hospital has to pay more salaries for them, but unknowing whether the outcome can overweigh its cost. Therefore, this group of studies was to detect the economic value of nursing education. On the one hand, evidence added by empirical study, they have found increasing the baccalaureate nurses' (BSN) dose to $80 \%$ for every patient could potentially result in $\$ 5.6$ million in savings annually that would more than offset annual costs of about
\$1.8 million in increased salaries associated with BSN qualifications. ${ }^{19}$ On the other hand, management department tries to detect more efficient way to quantify the value that nursing professional development brings to the organization ${ }^{20}$ and explored more undiscovered resource to assist nurses. For example, they found providing training curricula to non-nursing staff to provide feeding assistance for nutritionally at-risk nursing home $(\mathrm{NH})$ residents is cost-effective and has a positive effect on residents' between-meal intake. ${ }^{21}$

\subsection{Exploration of EE research in nursing field}

Classifying this group of studies is difficult because it included the terms, such as "health care," "Quality Assurance, Health Care," and "Nursing Care," which lack specific content. But we can think that the goal of EE is to improve the quality of health care. Therefore, we defined this topic according to the terms "nursing research" and "state medicine." Mark advocated using cost-effectiveness analysis for nursing research and believed the information provided by CEA will be an important additional component of the evidence produced by nursing research. ${ }^{22}$ Wendy systematically reviewed papers about EEs in nursing-relevant research performed in the United States between 1997 and 2015. She found that evaluation indicators in these studies are not standardized and EEs have not been fully utilized in US nursing-related studies over the past two decades. ${ }^{23}$ Studies in this group gave us the importance of EE in nursing research and we should correctly use economic analytical tools in researching.

\subsection{Nursing EE on care for neoplasm patient}

New drugs and treatment improved the survival rates of various cancers, resulting in the soaring costs of innovative technique and service needs to the increasing number of cancer survivors. ${ }^{24,25} \mathrm{EE}$ was served to 
find cost-effective care program or screening project for survivors. For example, Gordon found a telephonedelivered multiple health behavior change intervention that costs less but can improve physical activity, diet, and weight management. ${ }^{26}$ Groton selected colonoscopy bowel preparation protocols for colorectal cancer patients based on effectiveness, tolerability, and cost. ${ }^{27}$

\subsection{Nursing EE on extension of nurse function}

The topic of this group is to investigate the economic value and clinical outcome by expanding nurse's function. Two terms "Nurse Clinicians" and "Nurse Practitioners" can be seen in this group, they are considered advanced practice nurse, who has acquired the expert knowledge base, complex decision-making skills, and clinical competencies for expanded practice..$^{28}$ They expanded their function in an alternative role for physician and complementary role for registered nurse. ${ }^{29}$ Therefore, EE was produced to test whether they really could help to relieve workforce shortage, reduce cost, and improve care quality. At the same time, systematic review revealed that studies about the cost-effectiveness of advanced nurse are of poor methodological quality, indicating that higher quality studies are required..$^{30}$ From the terms "Patient Care Team" and "Case Management," we can see a new nursing model is developing. "Primary Health Care," "Home Care Services," and "Community Health Nursing" showed us that nursing workplace is not restricted in hospital and their role is extended to community and home. These new care models need economic analysis to evaluate their effectiveness, so they become a study topic in EE.

\section{References}

1. Lämås $K$, Willman $A$, Lindholm $L$, Jacobsson $C$. Economic evaluation of nursing practices: a review of literature. Int Nurs Rev. 2009;56:13-20.

2. Drummond MF, Sculpher MJ, Torrance GW, et al. Methods for the economic evaluation of health care programme. Third edition. Oxford: Oxford University Press. 2005.

3. Rudmik L, Drummond M. Health economic evaluation: important principles and methodology. Laryngoscope. 2013;123:1341-1347.

4. Elia M, Parsons EL, Cawood AL, et al. Costeffectiveness of oral nutritional supplements in older malnourished care home residents. Clin Nutr. 2018;37:651-658.

5. Franzini L, Sail KR, Thomas EJ, et al. Costs and cost-effectiveness of a telemedicine intensive care unit program in 6 intensive care units

\section{Conclusions}

Through co-word cluster analysis, we found the hotspots of research in nursing EE focus on the following topics: skin administration, infection prevention, education program, nurse education and management, EE research, neoplasm patient, and extension of nurse function. Although the amounts of papers are increasing, the quality of study is not promising. Therefore, further study may be required to detect nurses' knowledge of economic analysis method and their attitude to apply it into nursing research. Schools in developed countries have already set up some nursing economics courses, but the coverage rate is not high. Maybe that is the main reason for underutilization of EE in nursing research and the malpractice to use it. In the future, it might be possible to set up more nursing economics courses in more schools or hospitals.

\section{Limitation}

We used filter function in PubMed to limit the journal category in nursing, but probably excluded some related papers published in other types of journals, which may lead to a small bias in our results.

\section{Ethics approval}

Not declared.

\section{Conflicts of interest}

All contributing authors declare no conflicts of interest. in a large health care system. J Crit Care. 2011; 26:1-6.

6. Simmons SF, Hollingsworth EK, Long EA, et al. Training nonnursing staff to assist with nutritional care delivery in nursing homes: a cost-effectiveness analysis. J Am Geriatr Soc. 2017;65:313-322.

7. Huang M, Neveol A, Lu Z. Recommending MeSH terms for annotating biomedical articles. J Am Med Inform Assoc. 2011;18:660-667.

8. Theodosiou T, Vizirianakis IS, Angelis L, et al. MeSHy: mining unanticipated PubMed information using frequencies of occurrences and concurrences of MeSH terms. J Biomed Inform. 2011;44:919-926.

9. Lei $C$, Wei L, Lei $Y$, et al. Development of a text mining system based on the co-occurrence of bibliographic items in literature databases. New Tech Library Info Service. 2008;24:70-75 (in Chinese). 
10. Qinglan S. The demarcation of high - and low - frequency terms and ways of estimating the frequency of terms. J Library Science in China. 1992;18:78-81 (in Chinese).

11. Demarré $L$, Verhaeghe $S$, Annemans $L$, et al. The cost of pressure ulcer prevention and treatment in hospitals and nursing homes in Flanders: a cost-ofillness study. Int J Nurs Studies. 2015;52:1166-1179.

12. Tuffaha HW, Roberts S, Chaboyer W, et al. Costeffectiveness analysis of nutritional support for the prevention of pressure ulcers in high-risk hospitalized patients. Adv Skin Wound Care. 2016;29:261-267.

13. Cutting KF. The cost-effectiveness of a novel soluble beta-glucan gel. J Wound Care. 2017; 26: 228-234.

14. Anderson DJ, Pyatt DG, Weber DJ, et al. Statewide costs of healthcare-associated infections: estimates for acute care hospitals in North Carolina. Am J Infection Control. 2013; 41: 764-768.

15. Gidengil CA, Gay C, Huang SS, et al. Costeffectiveness of strategies to prevent methicillinresistant Staphylococcus aureus transmission and infection in an intensive care unit. Infect Control Hosp Epidemiol. 2015;36:17-27.

16. Mckinnell JA, Bartsch SM, Lee BY, et al. Cost-benefit analysis from the hospital perspective of universal active screening followed by contact precautions for methicillin-resistant Staphylococcus aureus carriers. Infect Control Hosp Epidemiol. 2015;36:2-13.

17. Mash R, Kroukamp R, Gaziano T, et al. Costeffectiveness of a diabetes group education program delivered by health promoters with a guiding style in underserved communities in Cape Town, South Africa. Patient Educ Couns. 2015;98:622-626.

18. Agren SSEL, Davidson T, et al. Cost-effectiveness of a nurse-led education and psychosocial programme for patients with chronic heart failure and their partners. J Clin Nurs. 2013;22:2347-2353.

19. Yakusheva O, Lindrooth $R$, Weiss M. Economic evaluation of the $80 \%$ baccalaureate nurse workforce recommendation: a patient-level analysis. Med Care. 2014;52:864-869.

20. Garrison E, Beverage J. Implementing a process to measure return on investment for nursing professional development. J Nurses Prof Dev. 2018; 34: 8-11.

21. Simmons SF, Hollingsworth EK, Long EA, et al. Training nonnursing staff to assist with nutritional care delivery in nursing homes: a cost-effectiveness analysis. J Am Geriatr Soc. 2017;65:313-322.

22. Bensink ME, Eaton LH, Morrison ML, et al. Cost effectiveness analysis for nursing research. Nurs Res. 2013;62:279-285.

23. Cook WA, Morrison ML, Eaton LH, et al. Quantity and quality of economic evaluations in U.S. nursing research, 1997-2015: a systematic review. Nurs Res. 2017;66:28-39.

24. Roberts KB, Soulos PR, Herrin J, et al. The adoption of new adjuvant radiation therapy modalities among Medicare beneficiaries with breast cancer: clinical correlates and cost implications. Int J Radiat Oncol Biol Phys. 2013;85:1186-1192.

25. Rishel CJ. Financial savvy: the value of business acumen in oncology nursing. Oncology Nursing Forum. 2014;41:324-326.

26. Gordon LG, Patrao T, Kularatna S, et al. A telephone-delivered multiple health behaviour change intervention for colorectal cancer survivors: making the case for cost-effective healthcare. Eur $J$ Cancer Care (Engl). 2015;24:854-861.

27. Groton M, Fisher MJ, Speroni KG, et al. A prospective, randomized, single-blind study evaluating the effectiveness, tolerability, and cost of colonoscopy bowel preparations. Gastroenterol Nurs. 2015;38:31-41.

28. Lopatina E, Donald F, Dicenso A, et al. Economic evaluation of nurse practitioner and clinical nurse specialist roles: A methodological review. Int J Nurs Studies. 2017;72:71-82.

29. Donald F, Kilpatrick K, Reid K, et al. A systematic review of the cost-effectiveness of nurse practitioners and clinical nurse specialists: what is the quality of the evidence? Nurs Res Pract. 2014;2014:1-28.

30. Marshall DA, Donald F, Lacny S, et al. Assessing the quality of economic evaluations of clinical nurse specialists and nurse practitioners: a systematic review of cost-effectiveness. Nursingplus Open. 2015;1:11-17. 